\title{
Effect of photonic flash annealing with subsequent compression rolling on the topography, microstructure and electrical performance of carbon-based inks
}

\author{
Sarah-Jane Potts ${ }^{1, *}$ (D), Yin Cheung Lau ${ }^{1}$ (D), Thomas Dunlop ${ }^{1}$ (D), Tim Claypole ${ }^{1}$ (D), and \\ Chris Phillips ${ }^{1}$ (1) \\ ${ }^{1}$ College of Engineering, Swansea University, Bay Campus, Crymlyn Burrows, Swansea SA1 8EN, UK
}

Received: 12 November 2018

Accepted: 18 February 2019

Published online:

27 February 2019

(C) The Author(s) 2019

\begin{abstract}
Binders used in screen-printed carbon-based inks are typically non-conductive. Photonic annealing and subsequent compression rolling have therefore been employed to remove binder and consolidate the conductive particles. Using this method, screen-printable carbon inks containing graphite only, graphite nanoplatelets and a combination of graphite and carbon black were assessed. Photonic annealing leads to the degradation of the polymer binder separating the carbon morphologies, with subsequent compression rolling leading to significant reductions in print film thickness, roughness and improvements in particle orientation. Both processes lead to electrical performance enhancement for all printed inks assessed. The process was most effective for single graphitic morphologies with large gaps between conductors. These saw significant improvements, with reductions in electrical resistivity from 1.91 to $0.23 \Omega \mathrm{cm}$ for the graphite ink. The mixed carbon ink saw smaller but still significant improvements in print roughness and resistivity, from 0.037 to $0.019 \Omega \mathrm{cm}$. Therefore, these postprocesses could widen the applications of common, lowcost carbon morphologies in screen printing inks.
\end{abstract}

\section{Introduction}

Screen-printed carbon-based pastes and inks are extensively used in a wide range of printed electronics applications including resistive heater panels [1-3], electrochemical sensors [4,5] pressure sensors [6], printed batteries [7] and supercapacitors [8] as well as photovoltaic applications including perovskite and dye-sensitised solar cells [9-12].
Graphite, carbon black and graphite nanoplatelets (GNPs) are the primary forms of carbon used in a large proportion of these devices in industry. This is due to their ease of use and economic advantage over other commercial conductive materials, such as silver and more recent derivatives of carbon such as graphene and carbon nanotubes (CNTs).

Consequently, research has been conducted into identifying the effects of modifying the ink

Address correspondence to E-mail: 654486@swansea.ac.uk 
formulations of graphite and carbon black-based inks. These include investigating the effect of the solvents used in the ink on the drying rate of the prints as well as its subsequent effects on film conductivity and process consistency [13]. Studies have also been conducted to assess the effects of the number of printed layers [1] on the electrical performance of the carbon electrodes, as well as the effects of different particle loadings [14] and ratios of the different carbon forms [13, 15]. These are improvements to the inks which have been quite well established industrially, such as in the blood glucose sensor industry. Despite these improvements, significant gains in conductivity might not be possible through formulation alone. In the case of altering the ratios of different carbon forms, it was found that the small carbon black particles were able to fill the gaps between the large graphite flakes [13]. Therefore, identifying methods which could be used to reduce the physical size of these gaps using postprocessing methods rather than alterations to the print formulation might also help drive further improvements to the print performance. This could enable print topography and conductivity to be enhanced without changing the ink formulation, which could otherwise compromise the quality of dispersion, shelf life and print performance for these common forms of carbon-based inks. From an electrochemical perspective, the binders in inks can block the active surfaces and thus result in slower reaction kinetics [16], making methods capable of reducing the binder in the print without compromising performance desirable. Postprocessing methods which have shown promise in the literature include photonic flash annealing followed by subsequent compression rolling.

Photonic methods, in the form of intensive pulsed light (IPL) sintering and annealing, have been used to provide a rapid burst of high energy to heat materials such as metal nanoparticles and copper oxide. This allows them to sinter and become conductive, and crucially, it can heat the print to high temperatures without damaging low-cost substrates such as polymer films or paper $[17,18]$, providing opportunities for low-cost plastic electronics. IPL sintering and annealing have been used in a range of printed electronics such as photovoltaic applications [19-21], where photonic annealing has been identified as a lower-cost alternative to high-energy, thermal processes. This enables a higher throughput along with improvements in the surface roughness, edge resolution and conductivity of the prints. It has also been used to enhance the electrical performance of RFID (radio frequency identification) tags $[22,23]$ and other forms of printed electronics [24, 25]. Arapov et al. [26] used photonic annealing to reduce the resistance of printed binder-based graphene inks from $200 \Omega \square^{-1}$ after thermal drying at $100{ }^{\circ} \mathrm{C}$ for $5 \mathrm{~min}$ (based on $6 \mu \mathrm{m}$ dry film thickness) to $40 \Omega \square^{-1}$ after photonic annealing.

Despite improvements in electrical performance, it was noted that photonic annealing also resulted in a reduction in layer adhesion and cohesion. The degradation of the binder caused a decrease in the number of contact points between the graphene sheets and therefore a loss of structural integrity [26]. To counteract this disruption, Arapov et al. used compression rolling to improve the structural integrity of the prints, as well as provide further improvements to resistivity, down to $20 \Omega \square^{-1}$, along with a reduction in film thickness and surface roughness from $6 \mu \mathrm{m}$ and $0.945 \mu \mathrm{m}$, respectively, after thermal drying to $1.7 \mu \mathrm{m}$ and $0.065 \mu \mathrm{m}$, respectively, after photonic annealing followed by compression rolling [26]. Compression rolling alone has also been investigated as a possible postprocessing method for a range of applications including radio frequency identification (RFID) antenna and other graphene-based prints and coatings, which in these cases were binder-free, where it produced improvements in the electrical and thermal conductivity of the devices due to better flake alignment [26-29]. There are also reports of compression rolling leading to improvements in the electrochemical performance of screen-printed lithium-ion batteries with electrodes consisting of graphite [30-32] and amorphous carbon [33]. In these cases, the compression rolling process has been found to reduce the roughness of the electrode by compressing the graphite flakes to become orientated with the basal plane, parallel to the current connector [32]. This leads to increases in the electrode's density and a reduction in porosity which enables improvements in the ionic and electrical conductive pathways [30, 31].

Although these analyses assess the effect of photonic annealing and compression rolling on single morphologies of carbon, these processes could also provide an effective method for improving the electrical performance of inks containing a range of carbon morphologies without having to change the ink formulations. Therefore, this paper investigated the 
effect of photonic annealing and compression rolling on inks containing a range of common, low-cost forms of carbon. Inks were made with the following carbon morphologies dispersed in solvent and polymeric binder: (1) graphite only, (2) graphite nanoplatelets (GNPs) only and (3) graphite with carbon black. The inks were manufactured, screen-printed, thermally dried and then posttreated. The effects of posttreatment were established using topography and resistance measurements of both printed solid areas and printed lines, as well as scanning electron microscopy (SEM) imaging after each of the stages. Thermogravimetric analysis (TGA) tests were also conducted to establish the equivalent temperatures and durations required to remove the binder using traditional thermal drying methods.

The effect of compression rolling on inks containing binder in comparison with those which have had their binder removed has also not been fully assessed. To establish whether the removal of binder is required to enable optimisation of print conductivity, the graphite only ink was used to assess the effect of compression rolling both with and without prior photonic annealing. These methods could provide a feasible technique for enhancing the print consistency and conductivity of low-cost carbon inks, which could lead to improvements in printed carbon electrodes for a host of devices, without increasing the cost or practicality of the ink formulation.

\section{Materials and methods}

\section{Ink formulation}

Inks with graphite only, graphite nanoplatelets (GNP) only and a combination of graphite with carbon black were made for the study. Materials used were as follows: graphite (Timrex ${ }^{\circledR}$ SFG15, Imerys Graphite and Carbon; typical D90 $17.9 \mu \mathrm{m}$ according to manufacturer); carbon black (TIMCAL ENSA$\mathrm{CO}^{\mathrm{TM}} 250 \mathrm{G}$ Conductive Carbon Black Compounds; with diameter distribution from 20 to $50 \mathrm{~nm}$ and specific surface area of $65 \mathrm{~m}^{2} \mathrm{~g}^{-1}$ according to the manufacturer); and graphite nanoplatelets (GNPs) functionalised with oxygen $\left(\mathrm{GNP}-\mathrm{O}_{2}\right.$ produced by Haydale Ltd. with average flake diameter around $5 \mu \mathrm{m}$ ). All inks used the same vinyl resin base (with $15 \%$ by weight dry polymer, VINNOL (Wacker Chemie AG) in 4-hydroxy-4-methylpentan-2-one (boiling point $\left.166^{\circ} \mathrm{C}\right)$ ) but with different loadings to suit processing and printing characteristics.

The graphite- and GNP-based inks were produced with $22.5 \%$ carbon concentrations (i.e. $22.5 \%$ carbon and $77.5 \%$ polymer dispersion by mass) as this was found to produce a good compromise between print quality and conductivity. Higher particle loadings were found to produce thicker and rougher print films with minimal improvements in line and sheet resistance and increases in resistivity. The ink containing a combination of carbon black and graphite was formulated based on optimal loading and ratios described previously by Phillips et al. [13.]: $29.4 \%$ carbon concentration, with 2.6 parts graphite to 1 part carbon black. The mass contents of all inks are described in Table 1. When making the inks, the carbon materials were gradually added to the premade resin and stirred in by hand, with the carbon black added before the graphite in the case of the graphite and carbon black ink. The ink slurries were then left to wet overnight before three-roll milling. Milling was conducted with an EXAKT80E three-roll mill (EXAKT Advanced Technologies $\mathrm{GmbH}$ ) with the same processing conditions used for all inks as described previously [13].

\section{Printing methodology}

Printing was carried out on a DEK 248 flatbed screen printing machine using a polyester mesh with 61 threads per $\mathrm{cm}, 64 \mu \mathrm{m}$ thread diameter and 13-micron emulsion, 2-mm snap-off, 65-70 Shore A hardness diamond squeegee of $130 \mathrm{~mm}$ long with a 12-kg squeegee force and print/flood speeds of $70 \mathrm{~mm} \mathrm{~s}^{-1}$. The substrate was PET (polyethylene terephthalateMelinex ${ }^{\circledR}$ 339, DuPont Teijin Films (175 $\mu \mathrm{m}$ thickness) opaque white). The print image included a series of 25-mm-long lines of nominal widths from 100 to $700 \mu \mathrm{m}$ and a $45-\mathrm{mm}$ square solid patch for sheet resistance assessment. Printed samples were dried in a conveyer dryer at $100{ }^{\circ} \mathrm{C}$ for $5 \mathrm{~min}$.

\section{Print characterisation before and after processing}

Print topography, microscopy analyses and electrical characterisation were conducted after printing and after each stage of postprocessing. White light interferometry (NT9300, Veeco Instruments, Inc., Plainview, NY, USA) was used to measure a full three- 
Table 1 Ink formulations for graphite, GNP and combined graphite and carbon black-based inks

\begin{tabular}{llllll}
\hline Inks & Dry resin (\%) & Solvent (\%) & Graphite (\%) & Carbon black (\%) & GNP (\%) \\
\hline Graphite-based ink & 11.6 & 65.9 & 22.5 & - & - \\
GNP-based ink & 11.6 & 65.9 & - & - & 22.5 \\
Graphite and carbon black-based ink & 10.6 & 60 & 21.3 & 8.1 & - \\
\hline
\end{tabular}

dimensional surface profile over the edge of the solid print so that the printed ink film thickness could be evaluated. Five times magnification was used, giving a measurement area of $1.2 \mathrm{~mm}$ by $0.93 \mathrm{~mm}$ (at a resolution of $736 \times 480$ pixels with sampling at 1.67$\mu \mathrm{m}$ intervals). The ink film thickness was calculated as the average height of the substrate subtracted from the average height of the ink, excluding the print edges where there tended to be a lip or a decline in ink film thickness depending on the print orientation. A total 36 measurements were taken for each ink type/processing combination. This was obtained over three print samples with 12 evenly spaced points around the perimeter of the printed square for each print. Average surface roughness measurements (Sa) over the printed area were also taken away from the edges. The microstructure of the prints was assessed using a JEOL JSL 7800F FEG scanning electron microscope $(\mathrm{SEM})$ at $3000 \times$ magnification.

Printed line geometry was also assessed at five times magnification with the white light interferometer. $700 \mu \mathrm{m}$ nominal width lines were each measured in three evenly spaced points. Geometric features were assessed by taking discrete measurements over the 1.2-mm-long sections measured by the interferometer (736 measurements at $1.67-\mu \mathrm{m}$ intervals for each measured section). From this, the standard deviation in line width and thickness was calculated.

Sheet resistance measurements were taken on the printed $45 \times 45 \mathrm{~mm}$ squares using a four-point probe method. A SDKR-13 probe (NAGY Messsysteme $\mathrm{GmbH}$ ) with a tip distance of $1.3 \mathrm{~mm}$ was used with a Keithley 2400 digital multimeter with subsequent conversion to sheet resistance using the appropriate correction factor from the data table proposed by Smits [34]. Sheet resistances are displayed as measured (with the correction factor), and to account for the variation in film thickness after postprocessing, resistivities were also calculated as the product of sheet resistance and ink film thickness.
A total of 12 sheet resistance vales were taken across the centre of each printed square to account for any deviation in print quality or effects of compression across the area. Line resistance was measured using the same multimeter in two-point mode for $700 \mu \mathrm{m}$ nominal width lines.

\section{Postprocessing: photonic annealing and compression}

Photonic annealing was performed using a PulseForge ${ }^{\circledR} 1200$ (NovaCentrix, Austin, TX, USA). A preliminary study was used to optimise voltage, pulse pattern and duration to produce the greatest improvement in conductivity in the prints. The pulse envelope of $2 \mathrm{~ms}$ in length and $260 \mathrm{~V}$ was used to provide a total energy of $1.10 \mathrm{~J} \mathrm{~cm}^{-2}$ to the samples. A single pulse of high energy resulted in catastrophic ablation and delamination of the print due to rapid gasification of binders within the ink. Therefore, an envelope comprised of 10 micro-pulses and a duty cycle of $80 \%$ of total energy was used to raise the temperature of the printed features more gradually to remove the binders in a more controlled manner.

Using the NovaCentrix SimPulse thermal simulation package, the peak temperature of the ink during photonic annealing was estimated to be $307^{\circ} \mathrm{C}$. This was based on measured layer thickness and a database of thermal characteristics of the materials. As the carbon-based prints were black, and the PET substrate was opaque white, light absorbance in the visible spectrum was far greater for the print than it was for the substrate. This allowed the PulseForge to selectively heat the printed features to a very high temperature, whilst the unprinted area remained below its glass transition temperature and was not distorted.

Compression rolling was performed using a Durston DRM 150 Rolling Mill (W Durston Ltd, UK). The compression pressure could not be directly measured but was manipulated by setting the gap between the rollers. Gap sizes from 0.05 to $0.25 \mathrm{~mm}$ were 
assessed, and a gap size of $0.125 \mathrm{~mm}$ was found in preliminary studies to generate the greatest improvement in conductivity without causing warpage of the substrate.

To evaluate the relative effects of photonic annealing followed by compression and compression alone, samples of printed graphite ink were also compression rolled without prior photonic annealing.

\section{Thermogravimetric analysis of ink and binder}

Thermogravimetric analysis (TGA) was used to provide an indication of the temperatures required to reduce the amount of binder in the inks using thermal treatment. Samples of the resin binder (15\% polymer by mass) and graphite ink were analysed. Testing was carried out by a PerkinElmer STA 6000 with a temperature ramp to $500{ }^{\circ} \mathrm{C}$ over $50 \mathrm{~min}$ $\left(10{ }^{\circ} \mathrm{C} \mathrm{min}^{-1}\right)$ in a nitrogen atmosphere.

\section{Results}

\section{Printed solid area}

\section{Topography and microstructure of printed solid squares}

Topographies of the prints for the various ink systems are shown before and after photonic annealing with subsequent compression rolling in Fig. 1. The resulting film thickness and surface roughness data are shown in Fig. 2, and scanning electronic microscope images of the surfaces are shown for graphite only, GNP only and graphite-carbon black in Figs. 3, 4 and 5, respectively. Summary of data is also provided in Table 2 together with data for graphite print compression only. For all inks, the print surface was relatively rough after thermal drying alone, with evidence of features such as voids, agglomerations and 'mesh marking' due to the interaction of the ink with the mesh during deposition (Fig. 1a-c). The features of mesh marking correspond with the frequency of the mesh used (61 threads per $\mathrm{cm}$ ) and are more prevalent in higher-viscosity inks which do not settle and slump after deposition, as was the case with these inks.

Graphite only ink
Prior to postprocessing, the graphite ink had the highest ink film thickness of all the inks at $9.8 \mu \mathrm{m}$ and average surface roughness of $2.8 \mu \mathrm{m}$ (Fig. 2), with signs of mesh marking, agglomerations and pinholes. This high surface roughness is due to low-frequency effects from the mesh but also the relatively large graphite flakes seemingly randomly orientated in the print. Flakes were orientated at a range of angles, between $0^{\circ}$ and $90^{\circ}$ to the horizontal axis, with large gaps between flakes and only small sections of the flakes in contact with neighbouring flakes, as shown in the SEM images (Fig. 3a). When the graphite ink prints were compression rolled directly after thermal drying, there was a reduction in both film thickness and average surface roughness, down to $6.2 \mu \mathrm{m}$ and $0.9 \mu \mathrm{m}$, respectively. The SEM images (Fig. 3b) demonstrate that compression rolling alone resulted in a significant increase in the area of the flakes in contact with one another, with flakes becoming aligned with the horizontal plane and filling in gaps between flakes. However, some gaps between flakes remained.

Prior to compression, the graphite ink prints that were photonically annealed showed an initial increase in film thickness and surface roughness up to $13.5 \mu \mathrm{m}$ and $3.2 \mu \mathrm{m}$, respectively. These increases corresponded with a change in microstructure as shown in the appropriate SEM image (Fig. 3c). As with the print which had been thermally dried only, the flakes were seemingly randomly orientated with only small areas of graphite flakes in contact with their neighbours. However, after photonic annealing there were more flakes aligned towards the vertical plane and the texture and edges of the flakes and gaps became more apparent.

When photonic annealing was followed with compression rolling, there was reduction in both average film thickness and surface roughness down to $4 \mu \mathrm{m}$ and $1.2 \mu \mathrm{m}$, respectively. These reductions are due to the flakes becoming densely compacted together, as shown in the appropriate SEM image (Fig. 3d). This was apparent to a greater extent than for prints compression rolled without photonic treatment (Fig. 4b). This resulted in smaller and fewer gaps between the flakes and alignment of the flakes with the horizontal plane, producing a smoother and thinner print when compared with compression alone.

Graphite nanoplatelet only ink 




Figure 1 White light interferometry images of the edges of the printed squares for the printed graphite, GNP, graphite with carbon black inks both before posttreatment (images a to c, respectively)

Prior to postprocessing, the GNP ink had an average film thickness and surface roughness of $8.7 \mu \mathrm{m}$ and $0.8 \mu \mathrm{m}$, respectively (Fig. 2). Irregular edges were apparent in the print (Fig. 1b), but the roughness was the lowest of all the inks, most likely due to the smaller size of the GNPs when compared with graphite. As with the graphite flakes, the GNPs in the print were orientated seemingly randomly, with the nanoplatelets orientated at various angles to the substrate, and a relatively large number of voids

and after both photonic annealing and compression rolling (images $\mathbf{d}$ to $\mathbf{f}$, respectively) at five times magnification.

between the nanoplatelets, as shown in the SEM image (Fig. 4a). As with the graphite inks, after photonic annealing, there was an increase in both film thickness and surface roughness to $10.3 \mu \mathrm{m}$ and $1.0 \mu \mathrm{m}$, respectively. After subsequent compression rolling, this was followed by a reduction in both film thickness and surface roughness to $4.7 \mu \mathrm{m}$ and $0.5 \mu \mathrm{m}$, respectively, with significant reductions in mesh marking (Fig. 1e). SEM images of the photonic then compression treated prints showed that GNPs 

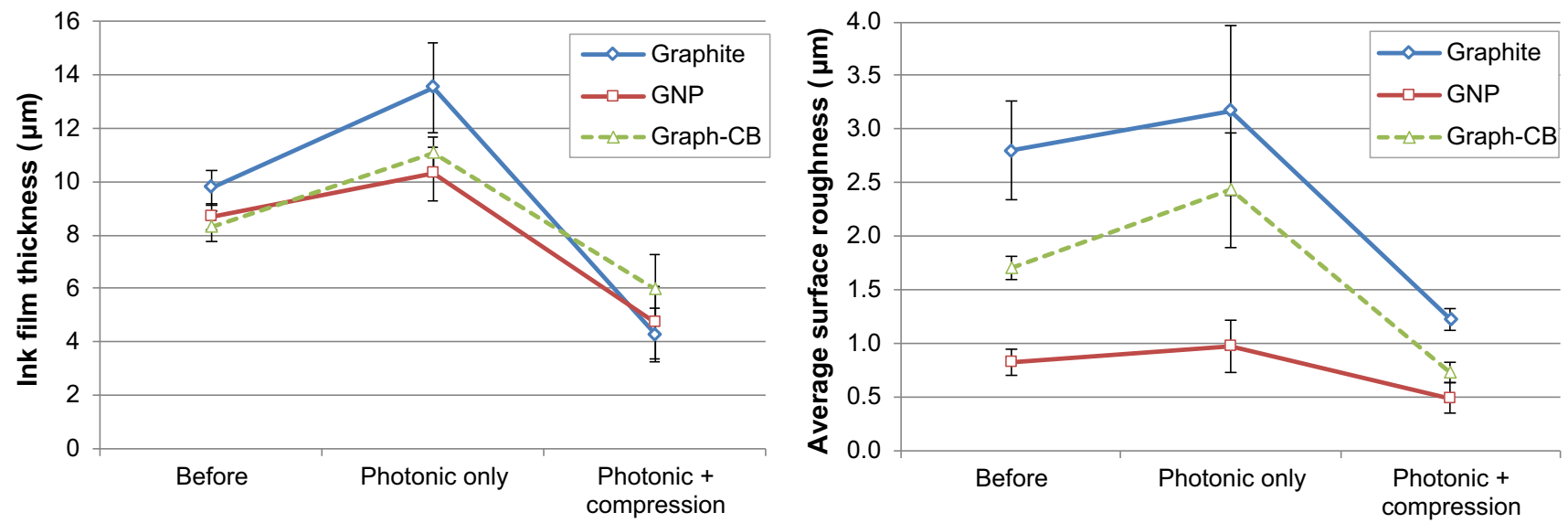

Figure 2 Film thickness (left) and average surface roughness ( $\mathrm{Sa}$ ) (right) for the various inks before and after photonic annealing and compression rolling (error bars show standard deviation).

Figure 3 Scanning electron microscope (SEM) images at 3000 times magnification showing the microstructure of the graphite-based inks which have received no postprocessing (top left), after compression rolling only (top right), after photonic annealing only (bottom left) and after both photonic annealing and compression rolling (bottom right).
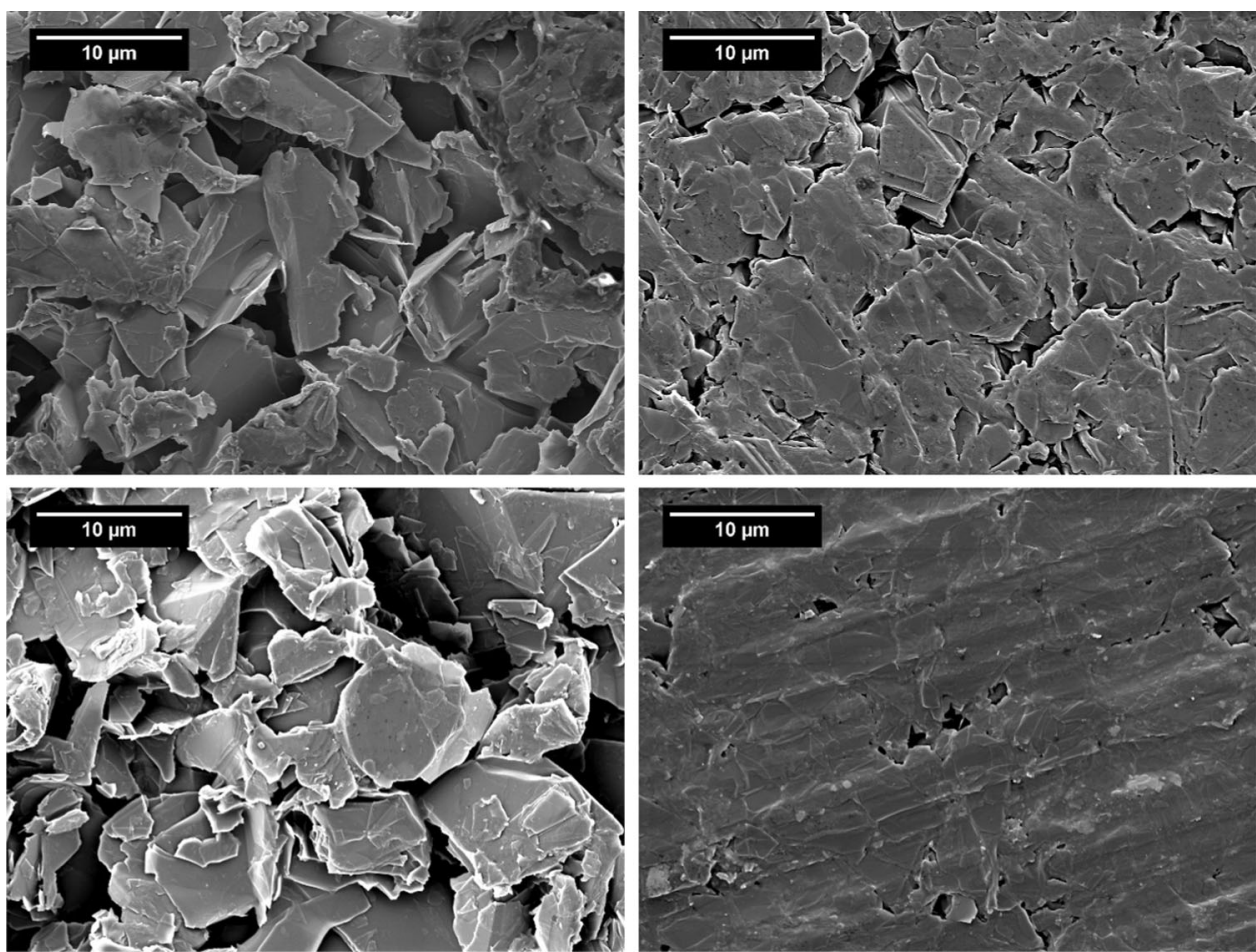

were aligned to the horizonal axis, lying flat with respect to the substrate (Fig. $4 \mathrm{~b}$ ). The boundaries between individual nanoplatelets were less clear than they were prior to postprocessing, and gaps between the GNPs appeared smaller.

Graphite and carbon black ink

The graphite and carbon black-based ink had an initial film thickness and surface roughness of $8.3 \mu \mathrm{m}$ and $1.7 \mu \mathrm{m}$, respectively (Fig. 2). Prior to processing, SEM images (Fig. 5) showed that, as with the graphite only ink, there were graphite flakes randomly orientated with small areas of the flakes in contact with one another. However, in this case the gaps between the flakes were partially filled with carbon black particles. Despite the carbon black acting as a filler material, the samples were relatively rough, with some peaks of the relatively large graphite flakes protruding out of the carbon black matrix, along with troughs between graphite flakes not filled with carbon black. As with the other inks, after photonic annealing only, there was an increase 


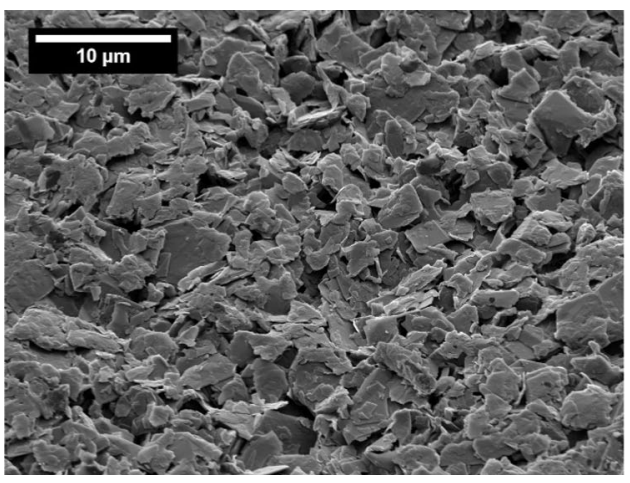

Figure 4 Scanning electron microscope (SEM) images at 3000 times magnification showing the microstructure of the graphite nanoplatelet (GNP)-based inks which have received no

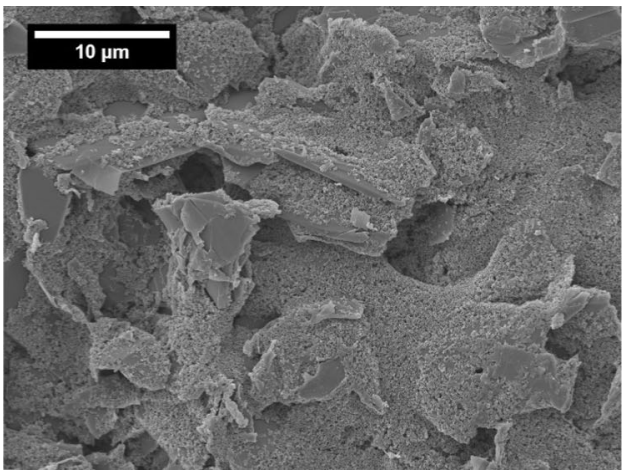

Figure 5 Scanning electron microscope (SEM) images at 3000 times magnification showing the microstructure of the graphite and carbon black-based inks which have received no postprocessing

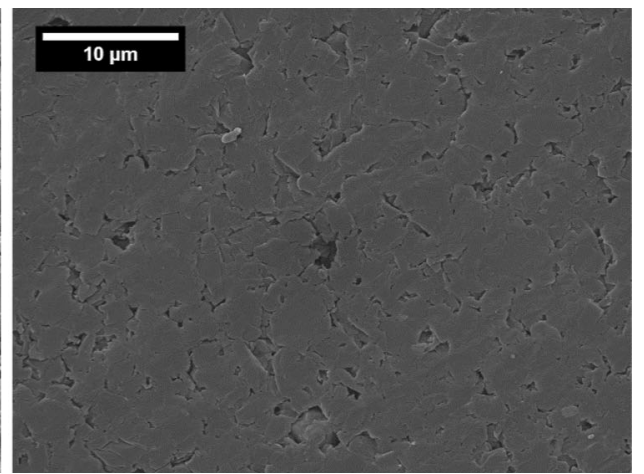

postprocessing (left) and which have undergone both photonic annealing and compression rolling (right).

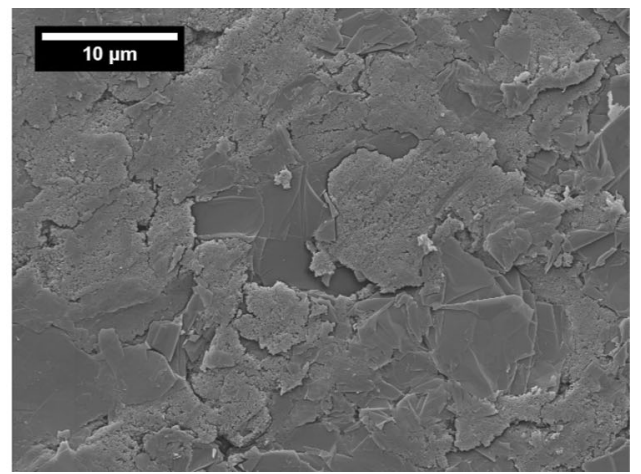

(left) and which have undergone both photonic annealing and compression rolling (right).

Table 2 Summary of ink film thickness and roughness of solid area data-standard deviation in parentheses

\begin{tabular}{|c|c|c|c|c|c|c|}
\hline & \multicolumn{3}{|c|}{ Ink film thickness $(\mu \mathrm{m})$} & \multicolumn{3}{|c|}{ Roughness of solid $(\mathrm{Sa} \mu \mathrm{m})$} \\
\hline & Graphite & GNP & Graphite-CB & Graphite & GNP & Graphite-CB \\
\hline Before & $9.78(0.64)$ & $8.68(0.51)$ & $8.33(0.57)$ & $2.80(0.46)$ & $0.82(0.12)$ & $1.70(0.11)$ \\
\hline Compression only & $6.21(1.20)$ & - & - & $0.90(0.2)$ & - & - \\
\hline Photonic only & $13.53(1.69)$ & $10.29(1.01)$ & $11.06(0.61)$ & $3.17(0.80)$ & $0.97(0.25)$ & $2.43(0.54)$ \\
\hline Photonic then compression & $4.27(1.01)$ & $4.71(1.34)$ & $5.94(1.32)$ & $1.22(0.10)$ & $0.49(0.14)$ & $0.72(0.10)$ \\
\hline
\end{tabular}

in the film thickness and surface roughness to $11.1 \mu \mathrm{m}$ and $2.4 \mu \mathrm{m}$, respectively. Subsequent compression reduced both film thickness and surface roughness to $5.9 \mu \mathrm{m}$ and $0.7 \mu \mathrm{m}$, respectively. After both photonic annealing and subsequent compression rolling, the graphite flakes became aligned to the horizontal plane with carbon black particles filling in gaps between the flakes, as shown in the SEM image (Fig. 5b). Despite these improvements, there appeared to be removal of carbon black from around the graphite particles.

\section{Sheet resistance of printed squares}

Sheet resistance and resistivity are compared before and after postprocessing for each of the inks in Figs. 6 and 7 , respectively. Summary of data is also provided in Table 3 with data for graphite print compression only. Sheet resistance is not affected by ink film 

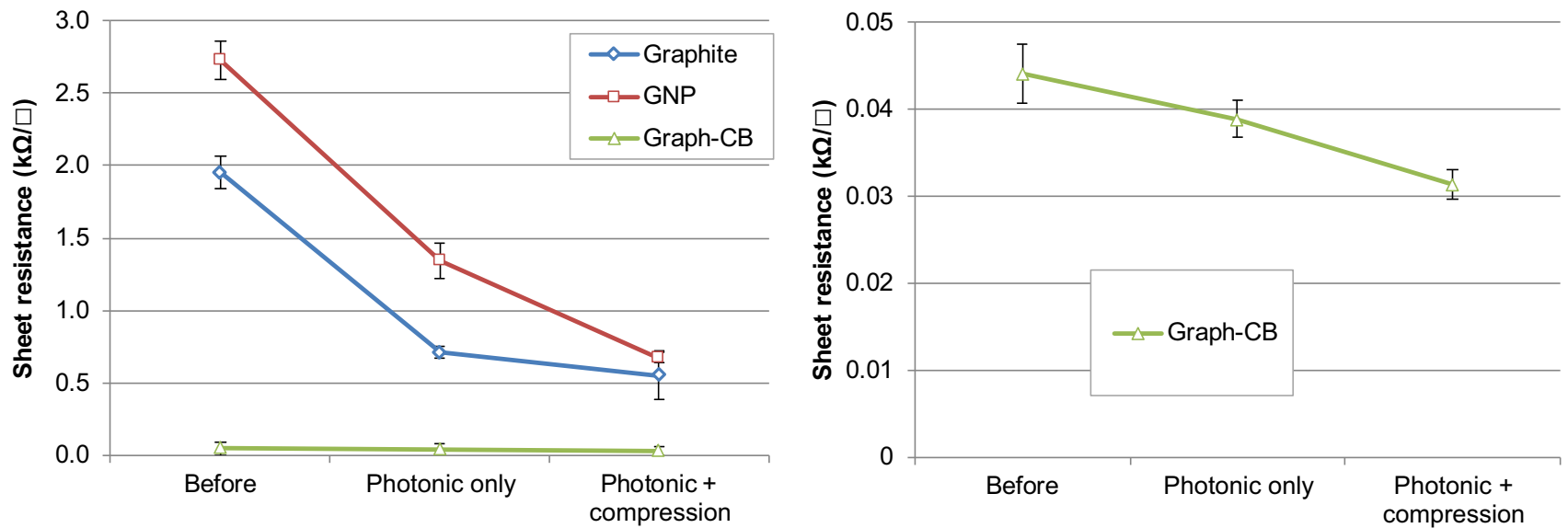

Figure 6 Sheet resistance of the printed squares before and after photonic curing and compression rolling for all inks (left) and graphite and carbon black-based ink only (right).
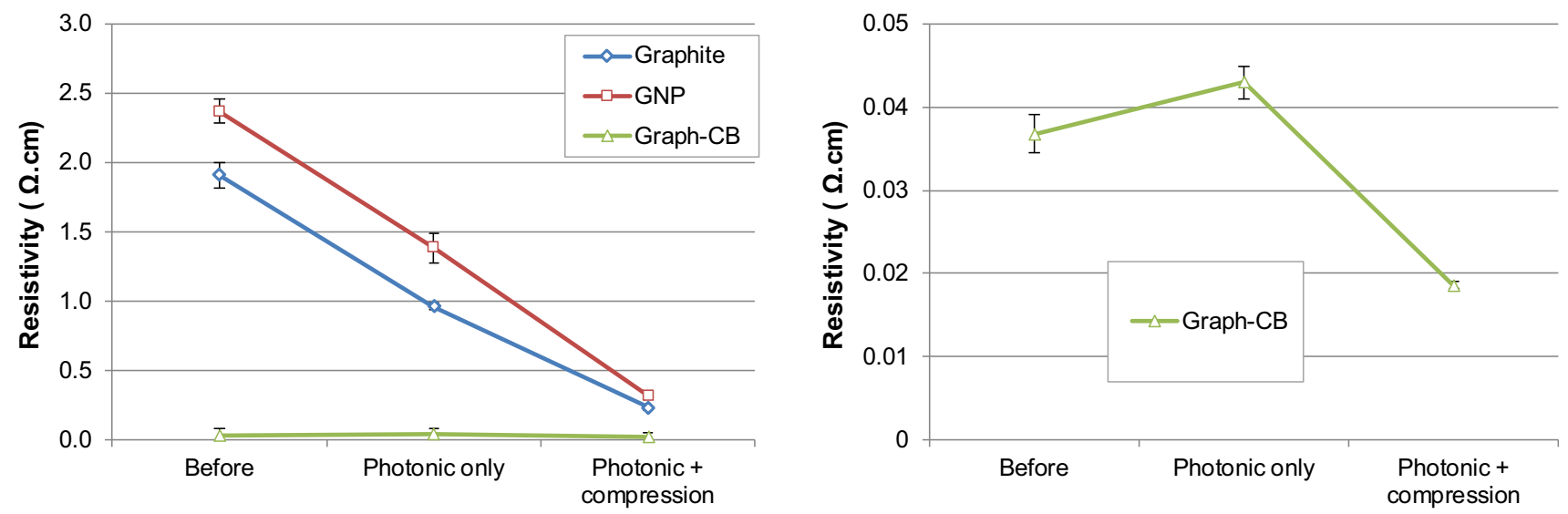

Figure 7 Resistivity of the printed squares before and after photonic curing and compression rolling for all inks (left) and graphite and carbon black-based ink only (right).

Table 3 Summary of sheet resistance and resistivity of solid area data - standard deviation in parentheses

\begin{tabular}{|c|c|c|c|c|c|c|}
\hline & \multicolumn{3}{|c|}{ Sheet resistance $\left(\mathrm{k} \Omega \square^{-1}\right)$} & \multicolumn{3}{|c|}{ Resistivity $(\Omega \mathrm{cm})$} \\
\hline & Graphite & GNP & Graphite-CB & Graphite & GNP & Graphite-CB \\
\hline Before & $1.95(0.11)$ & $2.73(0.14)$ & $0.044(0.003)$ & $1.91(0.09)$ & $2.37(0.09)$ & $0.037(0.002)$ \\
\hline Compression only & $1.49(0.30)$ & - & - & $0.74(0.10)$ & - & - \\
\hline Photonic only & $0.71(0.04)$ & $1.35(0.12)$ & $0.039(0.002)$ & $0.96(0.03)$ & $1.39(0.11)$ & $0.043(0.002)$ \\
\hline Photonic then compression & $0.55(0.17)$ & $0.68(0.04)$ & $0.031(0.002)$ & $0.24(0.02)$ & $0.32(0.004)$ & $0.019(0.000)$ \\
\hline
\end{tabular}

thickness, but the resistivity values allow the changing thickness of the ink to be taken into account. The graphite-based ink had an initial resistivity of $1.91 \Omega \mathrm{cm}$ after thermal drying only and displayed a substantial reduction in sheet resistivity after all postprocesses. In the case of the prints which were compression rolled directly after thermal drying, there was a reduction in resistivity down to $0.74 \Omega \mathrm{cm}$. The prints which were photonically annealed underwent a reduction to $0.96 \Omega \mathrm{cm}$, and following subsequent compression rolling, there was a further reduction to $0.23 \Omega \mathrm{cm}$ which represented a resistivity of around $12 \%$ of the initial value. Similarly, sheet resistance fell from $1.95 \mathrm{k} \Omega \square^{-1}$ to 1.49 , 
0.71 and $0.55 \mathrm{k} \Omega \square^{-1}$ for compression only, photonic annealing and photonic then compression, respectively. These changes are lower in magnitude as they do not take the change in ink film thickness into account. The compression rolling lead to an improvement in resistivity, most likely due to the increase in contact area between the conductive flakes and particles as they were aligned to the horizontal plain during compression.

The GNP ink had an initial resistivity of $2.37 \Omega \mathrm{cm}$ after thermal drying only which was slightly higher than that of the graphite ink. However, the GNP ink also showed a substantial reduction after both photonic annealing and subsequent compression rolling. There was reduction in resistivity to $1.38 \Omega \mathrm{cm}$ after photonic treatment, followed by a further reduction to $0.32 \Omega \mathrm{cm}$ after compression rolling. Both these posttreated resistivities were higher than for the graphite only ink. As with the graphite ink, this is likely to be due to the significant improvement in the flatness and contact area between the nanoplatelets described previously. Sheet resistances fell from an initial $2.73 \mathrm{k} \Omega \square^{-1}$ to 1.35 and $0.67 \mathrm{k} \Omega \square^{-1}$ for photonic annealing and photonic then compression, respectively.

The graphite and carbon black-based ink had an initial resistivity of $0.037 \Omega \mathrm{cm}$ which was substantially lower than the graphite- and GNP-based inks. Postprocessing only gave a small reduction in sheet resistance at each stage of processing. Compared with the other inks, there was a smaller but still significant improvement in resistivity upon processing, with a reduction in resistivity to $0.019 \Omega \mathrm{cm}$ after photonic annealing and subsequent compression rolling. However, there was an increase in resistivity to $0.042 \Omega \mathrm{cm}$ after photonic annealing only (although there was a reduction in sheet resistance). This is due to the photonic annealing process causing an increase in surface roughness and therefore average film thickness. Although this increase in film thickness occurred with the graphite and GNP inks also, the reduction in sheet resistance was more significant, thus counteracting this change in thickness. Nevertheless, the subsequent compression rolling lead to an overall improvement in the electrical performance as the graphite flakes were preferentially aligned towards the horizontal plane and the carbon black was compacted into the gaps between the flakes, leading to a greater area of conductive flakes and particles in contact. Sheet resistances fell from an initial $0.044 \mathrm{k} \Omega \square^{-1} \quad\left(44 \Omega \square^{-1}\right) \quad$ to $\quad 0.039$ and $0.031 \mathrm{k} \Omega \square^{-1}$ for photonic annealing and photonic then compression, respectively.

\section{Printed lines}

\section{Topography and resistance of printed lines}

The film thicknesses produced for the $700 \mu \mathrm{m}$ nominal width lines (of $25 \mathrm{~mm}$ in length) from each of the ink types are shown before and after postprocessing in Fig. 8. The resulting resistance values are shown in Fig. 9. The trends in both film thickness and resistance were similar to those produced for the printed squares for all inks. There was an increase in ink film thickness with photonic annealing, followed a reduction due to compression rolling, but line widths did not change significantly. Line resistances fell from 151.6 to $27.2 \mathrm{k} \Omega$ for graphite only, 317.6 to $64.9 \mathrm{k} \Omega$ for graphite only and 2.3 to $1.7 \mathrm{k} \Omega$ for graphite-carbon black.

\section{TGA of ink and binders}

Plots of mass percentage verses temperature from the TGA of the graphite-based ink and binder only are shown in Fig. 10. Mass loss began gradually from $30{ }^{\circ} \mathrm{C}$ and accelerated until peaking between $100{ }^{\circ} \mathrm{C}$ and $200{ }^{\circ} \mathrm{C}$. Both the graphite ink and binder alone then showed a plateau in weight loss at $200{ }^{\circ} \mathrm{C}$, when the majority of the solvent had been evaporated. This is supported by the $63 \%$ mass loss from the ink; which contained approximately $66 \%$ solvent in wet form, and $81 \%$ mass loss in the binder which initially

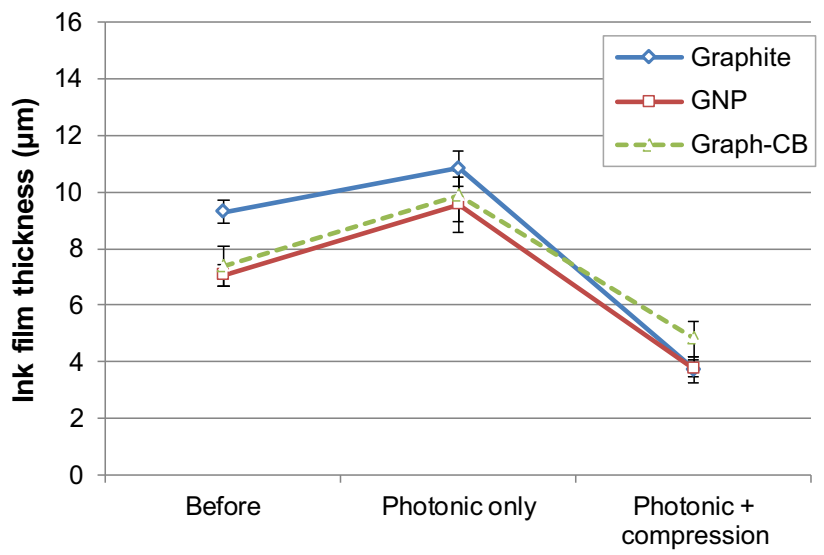

Figure 8 Ink film thickness for the printed $700 \mu \mathrm{m}$ nominal width lines before and after photonic curing and compression rolling. 

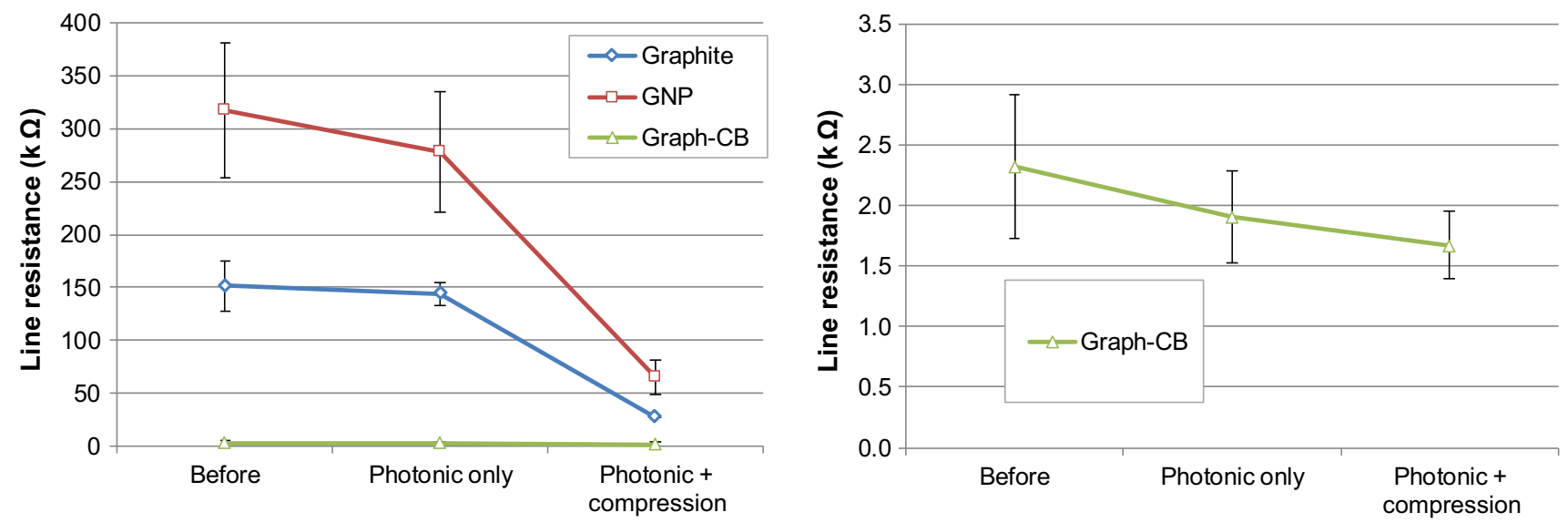

Figure 9 Resistance of the printed $700 \mu \mathrm{m}$ nominal width lines before and after photonic curing and compression rolling for all inks (left) and graphite and carbon black-based ink only (right).

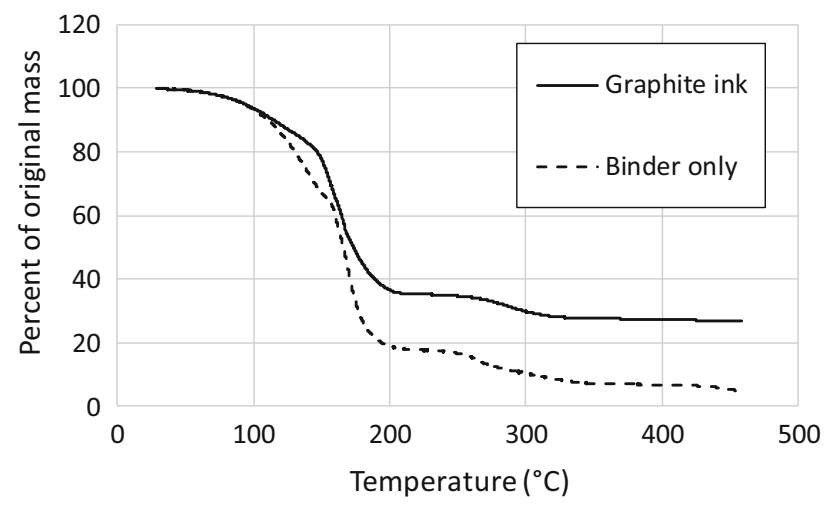

Figure 10 TGA of the graphite ink and binder only up to $500{ }^{\circ} \mathrm{C}$.

contained approximately $85 \%$ solvent. At approximately $250{ }^{\circ} \mathrm{C}$, the rate of mass loss started to increase before plateauing from around $330^{\circ} \mathrm{C}$ for the ink and $360{ }^{\circ} \mathrm{C}$ for the binder. By this point, all of the solvents will have likely been evaporated off and the observed mass loss most probably represents thermal degradation of the binder.

During thermal drying, a temperature of $100{ }^{\circ} \mathrm{C}$ was used so that structural damage and warping of the PET substrate did not occur. However, photonic treatment is able to rapidly heat the ink to a high temperature. This is sufficient to decompose the binder without degrading the substrate. The photonic annealing simulation software gave an indicative peak temperature of $307{ }^{\circ} \mathrm{C}$ which corresponded with a TGA mass loss of 71 and $90 \%$ for ink and dry resin, respectively. Although the thermal profiles of the two techniques are dissimilar, this suggests that photonically annealing the prints could lead to a significant reduction in the remaining resin in carbon inks.

\section{Discussion}

In the case of the graphite-based ink, reductions in surface roughness, film thickness and resistivity were apparent for samples which were compression rolled, both with and without prior photonic annealing. However, less significant improvements were seen for the samples which were compression rolled after drying alone, with a $68 \%$ reduction in surface roughness $(2.80-0.90 \mu \mathrm{m})$ and a $61 \%$ reduction in resistivity (1.91-0.74 $\Omega \mathrm{cm}$ ). For samples which were photonically annealed prior to compression rolling, there were greater reductions of $56 \%$ in surface roughness $(2.80-1.22 \mu \mathrm{m})$ and $88 \%$ in resistivity $(1.91-0.23 \Omega \mathrm{cm})$. TGA suggests that photonic annealing was able to reduce the binder in the print over a significantly shorter duration than would be required for traditional drying methods, where prolonged exposure to high temperatures would lead to warping of the polymer substrate [35]. This also complements the results by Secor et al. which found that photonic annealing was able to reduce the resistance of printed graphene inks by exceeding the threshold temperature required for the degradation of the polymer binder without damaging the substrate, as it was conducted on a short enough timescale. Their results found a reduction from $165 \Omega \square^{-1}$ when thermally dried to $135 \Omega \square^{-1}$ when photonically annealed [24]. This removal of insulating barriers between the graphite flakes could enable more direct contact points between the conductive flakes, and provide more space for the flakes to be compacted into during compression. On the other hand, for electrochemical applications, such as sensors, the 
rougher surface produced by photonic annealing only will provide a greater number of exposed edges and potentially increase the electron transfer rate [36, 37]. However, this must be weighed against durability and stability issues, as during photonic annealing the layered structure is interrupted and mechanical stability is reduced, resulting in poorer adhesion between the print and substrate. Subsequent compression rolling was used to consolidate the layer, and produce significantly smoother prints with the graphite flakes aligned to the horizonal plane, providing more substantial improvements in conductivity.

Similar improvements were shown in surface roughness and resistivity for the GNP-based inks, where there were reductions of $40 \%$ in surface roughness $(0.82-0.49 \mu \mathrm{m})$ and $86 \%$ in resistivity $(2.37-0.32 \Omega \mathrm{cm})$ after both photonic annealing and compression rolling. These resemblances are likely due to the similarities in the morphologies of the materials, i.e. layered planar structures which are initially randomly orientated. The random orientation of the GNPs provided large gaps between nanoplatelets from which binder could easily escape during photonic annealing and allow the GNPs to be compacted into during compression rolling. These changes in the print microstructure and improvements in the print conductivity, seen for the graphiteand GNP-based inks, are in line with the trends found for the graphene-based inks assessed by Arapov et al. that found a $80 \%$ reduction in sheet resistance after photonic annealing [26].

The graphite and carbon black ink was substantially more conductive than the GNP and graphitebased inks alone and therefore had lower potential for improvement in the conductive matrix. The carbon black particles acted as conductive bridges between the graphite flakes with the graphite-tocarbon black ratio optimised for conductivity by Phillips et al. [13]. This meant that there was less space for the graphite flakes to be flattened into during photonic annealing and subsequent compression rolling, when compared with the plain graphite and GNP inks. However, there were still improvements seen in the print topography which may have potential benefits; for example, Lee et al. suggest that polishing of an electrode surface will extend its working life and potentially make singleuse sensors reusable [16]. There were reductions of $59 \%$ in surface roughness $(1.7 \mu \mathrm{m}$ to $0.7 \mu \mathrm{m})$ for the graphite and carbon black-based prints with reductions of $49 \%$ in resistivity $(0.037-0.019 \Omega \mathrm{cm})$ after photonic annealing and subsequent compression rolling. However, the SEM images (Fig. 5) appear to show that some carbon black has been removed from the graphite flakes as a result of processing. So, although there is an overall improvement in print topography and electrical performance, due to an improvement in the area of carbon flakes and particles in contact with one another, it is done so whilst potentially removing the some of the uppermost carbon black particles. The high surface area and small size of these particles make them likely to be entrained in the vapour when the binder is degraded.

Overall, these postprocessing methods could provide a quick and simple technique to enhance conductivity with low-cost, easy-to-process materials. However, further improvements in conductivity could be made by using thicker printed layers, as found by Secor et al. for printed graphene inks, where the sheet resistance was found to reduce from around $135 \Omega \square^{-1}$ to around $20 \Omega \square^{-1}$, when increasing the number of printed layers from one to eight for samples which were photonically annealed [24]. Similar improvements were found for thermally dried samples, with a reduction from $165 \Omega \square^{-1}$ for one layer to around $30 \Omega \square^{-1}$ for eight layers, although the sheet resistance was consistently higher for all printed layers when compared with the photonically annealed counterparts. However, due to the increasing print thickness with number of passes, the pulse length and energy required for best results during photonic annealing would alter with deposit thickness and ink consistency [24].

\section{Conclusions}

This study has demonstrated that the photonic annealing and compression rolling can provide a suitable postprocessing method for improving the topography and electrical performance of carbonbased inks of a range of carbon morphologies. These processes both can be integrated into a roll to roll production of printed electronics and may be beneficial for a range of applications as well as potentially widening the range of uses for low-cost carbon inks, so that they can displace some of the applications using earth metal inks. Future work could be conducted to assess the effect of photonic annealing and 
compression rolling on the electrochemical performance of a range of redox couples for and electrochemical sensors, as well as battery electrodes, to assess whether these postprocessing methods can lead to advantages other than improvements in electrical conductivity.

\section{Acknowledgements}

The authors would like to acknowledge the funding from the European Social Fund via the Welsh Government, the Engineering and Physical Sciences Research Council (Grant reference: EP/L015099/1) and icmPrint that has made this research possible. SEM facilities were provided by the Swansea University AIM Facility; funded in part by the EPSRC (EP/M028267/1), the European Regional Development Fund through the Welsh Government (80708) and the Ser Solar project via Welsh Government. Compression roller equipment was provided by the Swansea University Materials Advanced Characterisation Centre (MACH1) funded through the ERDF A4B programme.

\section{Compliance with ethical standards}

Conflict of interest The authors declare that they have no conflict of interest.

Open Access This article is distributed under the terms of the Creative Commons Attribution 4.0 International License (http://creativecommons.org/ licenses/by/4.0/), which permits unrestricted use, distribution, and reproduction in any medium, provided you give appropriate credit to the original author(s) and the source, provide a link to the Creative Commons license, and indicate if changes were made.

\section{References}

[1] Philip B, Jewell E, Greenwood P, Weirman C (2016) Material and process optimization screen printing carbon graphite pastes for mass production of heating elements. J Manuf Process 22(1):185-191. https://doi.org/10.1016/j.j mapro.2016.03.001

[2] Park HK, Kim SM, Lee JS, Park JH, Hong YK, Hong CH et al (2015) Flexible plane heater: graphite and carbon nanotube hybrid nanocomposite. Synth Met
203(1):127-134. https://doi.org/10.1016/j.synthmet.2015.02. 015

[3] Serra N, Maeder T, Lemaire P, Ryser P, Maedera T, Lemaire $P$ et al (2009) Formulation of composite resistive pastes for micro-heater manufacturing. Procedia Chem 1(1):48-51

[4] Wang J, Tian B, Nascimento VB, Angnes L (1998) Performance of screen-printed carbon electrodes fabricated from different carbon inks. Electrochim Acta 43(23):3459-3465

[5] Morrin A, Killard AJ, Smyth MR (2003) Electrochemical characterization of commercial and home-made screenprinted carbon electrodes. Anal Lett 36(9):2021-2039

[6] Knite M, Teteris V, Kiploka A, Kaupuzs J (2004) Polyisoprene-carbon black nanocomposites as tensile strain and pressure sensor materials. Sens Actuators A Phys 110(1-3):142-149

[7] Tehrani Z, Korochkina T, Govindarajan S, Thomas DJ, Mahony JO, Kettle J et al (2015) Ultra-thin flexible screen printed rechargeable polymer battery for wearable electronic applications. Org Electron 26(1):386-394. https://doi.org/10. 1016/j.orgel.2015.08.007

[8] Tehrani Z, Thomas DJ, Korochkina T, Phillips CO, Lupo D, Lehtim S et al (2017) Large-area printed supercapacitor technology for low-cost domestic green energy storage. Energy 118(1):1313-1321

[9] Philip B, Jewell E, Worsley D (2016) The impact of solvent characteristics on performance and process stability of printed carbon resistive materials. J Coatings Technol Res 13(5):911-920

[10] Zhang L, Liu T, Liu L, Hu M, Yang Y, Mei A et al (2015) The effect of carbon counter electrodes on fully printable mesoscopic perovskite solar cells. J Mater Chem A 3(17):9165-9170

[11] Murakami TN, Ito S, Wang Q, Nazeeruddin MK, Bessho T, Cesar I et al (2006) Highly efficient dye-sensitized solar cells based on carbon black counter electrodes. J Electrochem Soc 153(12):A2255. https://doi.org/10.1149/1.2358087

[12] Imoto K, Takahashi K, Yamaguchi T, Komura T, Nakamura JI, Murata K (2003) High-performance carbon counter electrode for dye-sensitized solar cells. Sol Energy Mater Sol Cells 79(4):459-469

[13] Phillips C, Al-Ahmadi A, Potts S-J, Claypole T, Deganello D (2017) The effect of graphite and carbon black ratios on conductive ink performance. J Mater Sci 52(16):9520-9530. https://doi.org/10.1007/s10853-017-1114-6

[14] Jewell EH, Hamblyn SM, Claypole TC, Gethin DT (2013) The impact of carbon content and mesh on the characteristics of screen printed conductive structures. Circuit World 39(1):13-21. https://doi.org/10.1108/03056121311298918

[15] Oxfall H, Ariu G, Gkourmpis T, Rychwalski RW, Rigdahl M (2015) Effect of carbon black on electrical and rheological 
properties of graphite nanoplatelets/poly(ethylene-butyl acrylate) composites. Express Polym Lett 9(1):66-76

[16] Lee J, Arrigan DWM, Silvester DS (2016) Mechanical polishing as an improved surface treatment for platinum screen-printed electrodes. Sens Bio Sens Res 9:38-44. http s://doi.org/10.1016/j.sbsr.2016.05.006

[17] Kenfack A, Vemo BN, Ngoula F, Anyangwe FF, Chombong JK, Et Joseph Tchoumboué AMMT (2015) Comparison of laser and intense pulsed light sintering (IPL) for inkjetprinted copper nanoparticle layers. Sci Rep 5(8832):2-11. h ttps://doi.org/10.1038/srep08832

[18] Albrecht A, Rivadeneyra A, Abdellah A, Lugli P, Salmerón JF (2016) Inkjet printing and photonic sintering of silver and copper oxide nanoparticles for ultra-low-cost conductive patterns. J Mater Chem C. 4(16):3546-3554

[19] Druffel T, Dharmadasa R, Lavery BW, Ankireddy K (2018) Intense pulsed light processing for photovoltaic manufacturing. Sol Energy Mater Sol Cells 174(1):359-369

[20] Troughton J, Carnie MJ, Davies ML (2016) Photonic flashannealing of lead halide perovskite solar cells in $1 \mathrm{~ms}$. J Mater Chem A 4(1):3471-3476

[21] Galagan Y, Coenen EWC, Abbel R, Van Lammeren TJ, Sabik S, Barink M et al (2013) Photonic sintering of inkjet printed current collecting grids for organic solar cell applications. Org Electron 14(1):38-46. https://doi.org/10.1016/j. orgel.2012.10.012

[22] Amin Y, Chen Q, Zhen LR, Tenhunen H (2012) Design and fabrication of wideband archimedean spiral antenna based ultra-low cost "green" modules for RFID sensing and wireless applications. Prog Electromagn Res 130(1):241-256

[23] Turki BM, Abbel R, Barbosa S, Tate DJ, Oyeka D, Batchelor JC et al (2015) Inkjet printed paper based frequency selective surfaces and skin mounted RFID tags: the interrelation between silver nanoparticle ink, paper. J Mater Chem C 3(1):2132-2140

[24] Secor EB, Ahn BY, Gao TZ, Lewis JA, Hersam MC (2015) Rapid and versatile photonic annealing of graphene inks for flexible printed electronics communication. Adv Mater 27(1):6683-6688

[25] Kim H, Dhage SR, Shim D, Hahn HT (2009) Intense pulsed light sintering of copper nanoink for printed electronics. Appl Phys A 97(1):791-798

[26] Arapov K, Bex G, Hendriks R, Rubingh E, Abbel R, de With $\mathrm{G}$ et al (2016) Conductivity enhancement of binder-based graphene inks by photonic annealing and subsequent compression rolling. Adv Eng Mater 18(7):1234-1239

[27] Coscia U, Palomba M, Ambrosone G, Barucca G, Cabibbo M, Mengucci P et al (2017) A new micromechanical approach for the preparation of graphene nanoplatelets deposited on polyethylene. Nanotechnology 28(19):194001

[28] Malekpour H, Chang KH, Chen JC, Lu CY, Nika DL, Novoselov KS et al (2014) Thermal conductivity of graphene laminate. Nano Lett 14(9):5155-5161

[29] Huang X, Leng T, Zhang X, Chen JC, Chang KH, Geim AK et al (2015) Binder-free highly conductive graphene laminate for low cost printed radio frequency applications. Appl Phys Lett 106(20):203105

[30] El Baradai O, Beneventi D, Alloin F, Bongiovanni R, BruasReverdy N, Bultel Y et al (2016) Microfibrillated cellulose based ink for eco-sustainable screen printed flexible electrodes in lithium ion batteries. J Mater Sci Technol 32(6):566-572. https://doi.org/10.1016/j.jmst.2016.02.010

[31] Sheng Y, Fell CR, Son YK, Metz BM, Jiang J, Church BC (2014) Effect of calendering on electrode wettability in lithium-ion batteries. Front Energy Res 2(December):1-8. h ttps://doi.org/10.3389/fenrg.2014.00056

[32] Gnanaraj JS, Cohen YS, Levi MD, Aurbach D (2001) The effect of pressure on the electroanalytical response of graphite anodes and $\mathrm{LiCoO}_{2}$ cathodes for Li-ion batteries. J Electroanal Chem 516:89-102

[33] Wang C, Yi Y, Sastry AM, Shim J, Striebel KA (2004) Particle compression and conductivity in li-ion anodes with graphite additives. J Electrochem Soc 151(9):A1489-A1498

[34] Smits FM (1958) Measurement of sheet resistivities with the four-point probe. Bell Syst Tech J 37(3):711-718

[35] Farnsworth S, Schroder K (2012) Photonic curing for millisecond-drying of thin films. Spec Print Worldw 1(4):34-36

[36] Kadara RO, Jenkinson N, Banks CE (2009) Characterisation of commercially available electrochemical sensing platforms. Sens Actuators B Chem 138(2):556-562

[37] Fanjul-Bolado P, Hernández-Santos D, Lamas-Ardisana PJ, Martín-Pernía A, Costa-García A (2008) Electrochemical characterization of screen-printed and conventional carbon paste electrodes. Electrochim Acta 53(10):3635-3642

Publisher's Note Springer Nature remains neutral with regard to jurisdictional claims in published maps and institutional affiliations. 